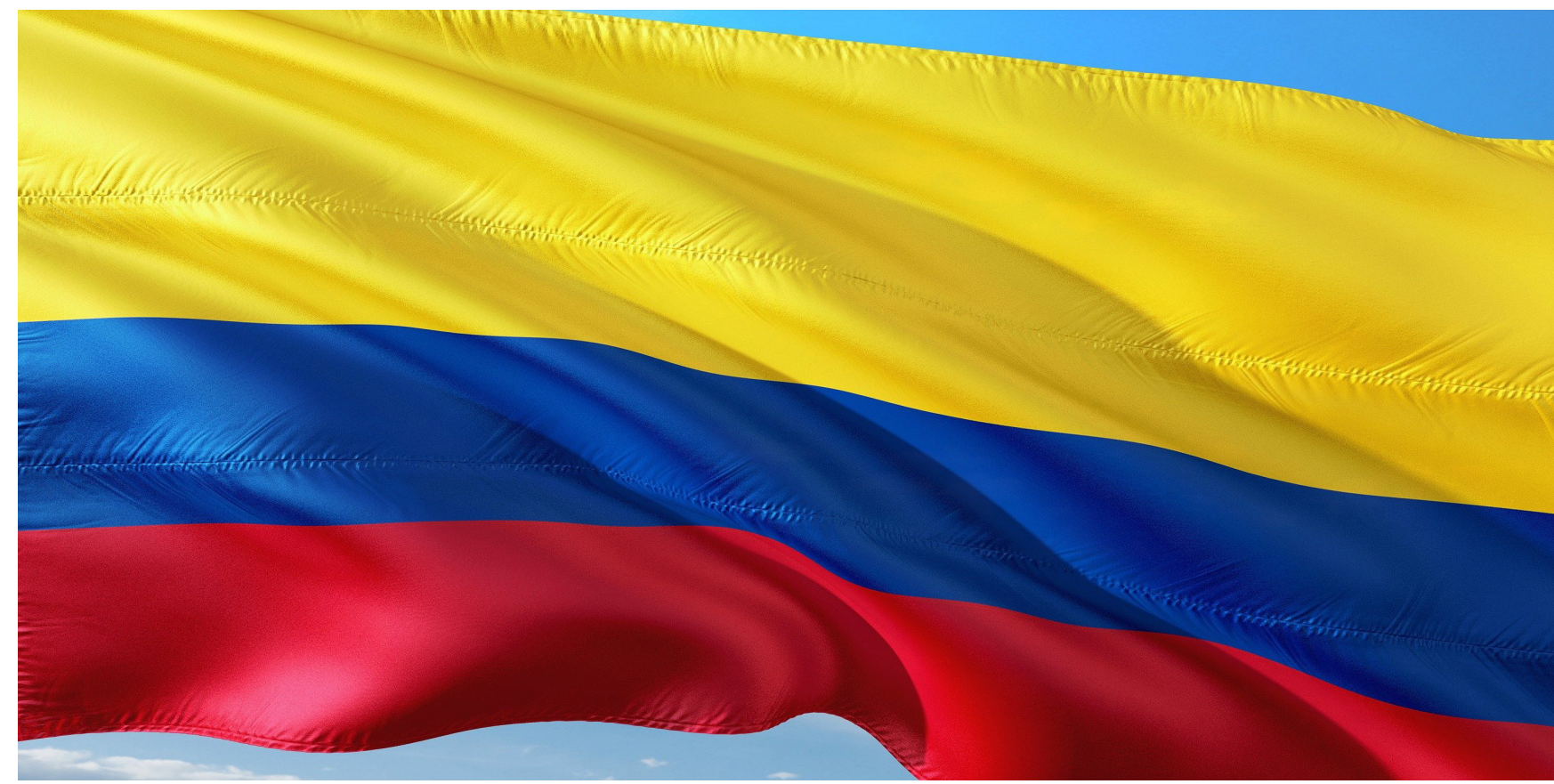

\title{
El sistema electoral de la cifra repartidora y la participación en política por parte de las Farc en el marco del postconflicto colombiano
}

\section{The electoral system of the number of voters and the participation in politics by the Farc in}

Mario Armando Echeverría-Acuña

Doctor (c) en Ciencias Sociales, Magíster en Derecho, Especialista en Derecho Constitucional y Derecho Administrativo,

mario.echeverria@unilibrectg.edu.co, Universidad Libre Cartagena,

Cartagena de Indias, Colombia

\section{María Alejandra Benítez-Hurtado}

Magíster en Derecho Administrativo, mariaa-benitezh@unilibre.edu.co, Integrante del Grupo de Investigación Filosofía del Derecho y Derecho

Constitucional, Universidad Libre Cartagena, Cartagena de Indias, Colombia 


\section{Resumen}

El presente trabajo pretende abordar el tema de la participación en política por parte de las FARC una se firmen los Acuerdos en La Habana, con el análisis de los sistemas electorales en especial el de la Cifra Repartidora, contemplado en el artículo 263A de nuestra Carta Política, el cual incluye el estudio de lo acordado en el Punto Segundo del Acuerdo General para la terminación del conflicto y del Acto Legislativo 01 del 2012, atinente al Marco Jurídico Para la Paz. Se hace evidente que las FARC buscan en la Mesa de diálogos tener un espacio político en el sistema y ser alternativa política válida de gobierno. La derecha tradicional debe compartir el poder o bien soportar el desgaste de la lucha guerrillera y de las organizaciones armadas del narcotráfico, algo impensable en la era de la globalización.

Palabras clave: Participación política, Democracia Participativa, Sistemas Electorales, Cifra repartidora, Régimen Electoral.

\section{Abstract}

This paper aims to address the issue of political participation by the FARC once the Agreements are signed in Havana, with an analysis of the electoral systems, especially that of the Cifra Repartidora, referred to in Article 263A of our Political Charter, which includes the study of what was agreed in Point Two of the General Agreement for the End of the Conflict and Legislative Act 01 of 2012, relating to the Legal Framework for Peace. It is evident that the FARC seeks to have a political space in the system and to be a valid political alternative to the government at the talks. The traditional right must share power or else endure the wear and tear of the guerrilla struggle and the armed drug trafficking organizations, something unthinkable in the era of globalization.

Keywords: Political participation, Participatory Democracy, Electoral Systems, proportional representation, Electoral System. 
El sistema electoral de la cifra repartidora y la participación en política por parte de las farc en el marco del postconflicto colombiano

86

\section{Introducción}

Podemos definir al Postconflicto como la etapa que sigue a la terminación de una situación de "guerra civil", donde en virtud de una situación de indefinición estratégica de índole militar, las fuerzas enfrentadas, las del estado y las que quieren cambiar la estructura de poder, negocian cambios político-económicos que permitan a las segundas, convertirse en fuerza política organizada, dejando las armas.

Comienza, una vez se incorporen a la Constitución Política los cambios citados o, se haga otra Constitución. Dura tanto como se estime que deba ser la transición y las fuerzas que se enfrentaron aprendan a convivir políticamente conforme a las normas constitucionales que resultaren del acuerdo. [1].

Esta precisión conceptual se establece a partir de las bases de Teoría Política sin las cuales lo que debemos entender por Constitución y Derecho Constitucional carecería de sentido; no perdamos de vista que la materia regulada por una Constitución es la organización y práctica de la política, es decir, el ejercicio del poder.

Referente a la participación en política, que es un punto acordado de la Agenda General para la terminación del conflicto, no se tenía certeza si las FARC serían luego de la dejación de armas un Movimiento Político o los ex combatientes participarían de forma individual en la esfera política, y sobre los dos supuestos escenarios, académicos propusieron diversas alternativas para garantizar el pleno y legítimo ejercicio de la política por parte de las FARC. Se tomaron referencias internacionales como las de Nicaragua - en donde se reformó la normatividad electoral, haciendo más equitativa el sistema de propaganda electoraly El Salvador -donde se estableció un nuevo código electoral y la creación de la figura del fiscal electoral [2].

Al hacerse público lo acordado en el punto dos de la agenda, si bien hubo avances, quedan bajo el margen de la duda ciertos aspectos atinentes a las reformas o cambios sustanciales necesarios para lograr una efectiva participación en política por parte de las FARC, como por ejemplo el abandono del sistema de la cifra repartidora, el cual ha impedido la supervivencia de una verdadera oposición en nuestro país.

Por lo anterior, y luego de una revisión exhaustiva de lo pactado en participación política, nos preguntamos ¿Cuáles son las cambios que deben efectuarse en el sistema electoral colombiano para garantizar la participación política de las FARC de cara a un eventual postconflicto?, defendiendo la tesis de que una vez se firme el acuerdo final es importante una reforma de la cifra repartidora puesto que en las condiciones actuales, un escenario anti-democrático como el que favorece este sistema de representación de los partidos políticos pone en riesgo la implementación exitosa de la intervención de las FARC como grupo político, arriesgándonos entonces a volver al punto que dio origen a este conflicto de más de medio siglo que hoy aspiramos terminar.

Metodológicamente la investigación es de carácter política-jurídica, aplicada, descriptiva y bibliográfica, de tipo cualitativa sobre el contenido y alcance que implique el objetivo antes dicho. Todo lo anterior porque la investigación dará solución a un problema actual, su descripción y su significado [3].

Una mirada al acuerdo sobre participación en política por parte de las Farc.

Siendo conscientes de cuán importante es la apertura democrática para la construcción 
de la paz y así culminar con el nexo entre el ejercicio de la política y la violencia, uno de los puntos producto del Acuerdo General para la terminación del conflicto y la construcción de una paz estable y duradera es el atinente a la Participación en política, buscando en el mismo sentido fortalecer la representación de las diferentes visiones e intereses de la sociedad y promover la participación ciudadana a través del fortalecimiento de las organizaciones y movimientos sociales, y el robustecimiento de los espacios de participación ciudadana [4].

En cuanto a los derechos y garantías para la oposición, se pactó el establecimiento del estatuto de la oposición, con el previo concurso de todos los partidos y movimientos sociales con personería jurídica, mientras que para las organizaciones y movimientos sociales y populares es necesario, no sólo garantizar el pleno ejercicio de derechos y libertades, incluyendo el de hacer oposición, sino también promover y facilitar los espacios para que tramiten sus peticiones [5].

Así mismo, se acordó la creación del Sistema Integral de Seguridad para el ejercicio de la política cuyo objeto es la protección de la vida y libertad de pensamiento de las personas; es un sistema que acoge en especial tanto a aquellas personas que producto de este proceso han abandonado las armas y ejercen legalmente la política, como a líderes de organizaciones y movimientos sociales y defensores de derechos humanos en situación de riesgo.

Como parte del pluralismo, se convino eliminar la obtención y conservación de la personería jurídica de los partidos y movimientos políticos del requisito de la superación de un umbral en las elecciones de Congreso; la creación de un modelo donde a los partidos con mejor práctica electoral en los ámbitos municipal, departamental y nacional se le reconozcan prerrogativas; la distribución ecuánime de los recursos a los partidos políticos con representación en el Congreso, con el aumento de esos fondos; y ampliación y consolidación de espacios de divulgación del programa político de los partidos o movimientos políticos con representación en el Congreso [6].

Para promover la participación electoral se acordó la creación de las Circunscripciones Transitorias Especiales de Paz en las regiones más golpeadas del conflicto, cuyos habitantes pueden elegir durante la fase de transición y de manera temporal, representantes adicionales con unas reglas especiales, estos candidatos deben pertenecer a la comunidad o que con ocasión de conflicto fueron desplazados y estén en proceso de retorno, y por consiguiente lograr la integración de las regiones afectadas por el conflicto. Siguiendo el mismo propósito se adoptó lo siguiente:

- Medidas para remover obstáculos y facilitar el ejercicio del derecho al voto, con especial énfasis en las poblaciones más vulnerables.

- Medidas para promover la transparencia en los procesos electorales.

- Adoptar medidas para garantizar mayor transparencia de la financiación de las campañas electorales.

- Llevar a cabo una reforma del régimen y de la organización electoral, con el objetivo de asegurar una mayor autonomía e independencia de la organización electoral [7].

Para la participación ciudadana a través de medios de comunicación comunitarios, institucionales y regionales quedó sentado la apertura de convocatorias para adjudicación de radio comunitaria, con énfasis en las zonas 
El sistema electoral de la cifra repartidora y la participación en política por parte de las farc en el marco del postconflicto colombiano

88

más afectadas por el conflicto y así promover la democratización de la información y del uso del espectro electromagnético disponible; en esa misma línea, la capacitación técnica de los trabajadores de los medios comunitarios, comunicadores y operadores, acompañado de financiación para la apertura de espacios en emisoras y canales institucionales y así divulgar el trabajo de las organizaciones y movimientos sociales, y de las comunidades en general [8].

Se adiciona a la avenencia el establecimiento del Consejo Nacional para la Reconciliación y la Convivencia, también en los órdenes territoriales, con la finalidad de promover la cultura de la reconciliación, convivencia, tolerancia y no estigmatización a través de la promoción de esta cultura, programas y la creación de una cátedra de cultura política para la reconciliación y la paz.

Por último, se le dio preminencia al papel de la mujer en el liderazgo política y una cultura de tolerancia y mutuo respeto; también a las veedurías y el control ciudadano en la implementación del Acuerdo Final, que se pondrá en marcha con el concurso de organizaciones especializadas e instituciones de educación superior, entre otros, que proveerán acompañamiento y asistencia técnica; todo esto acompañado de capacitaciones y creación de mecanismos de información [9].

Todo lo anterior, junto con los cuatro puntos restantes del Acuerdo General, debe ser aprobado por el pueblo colombiano en el plebiscito propuesto por el Ejecutivo, si este aprueba el examen de exequibilidad por parte de la Corte Constitucional.

Acto legislativo 01 de 2012 y participación política de las Farc.

El Acto legislativo 01 de 2012, denominado Marco Jurídico para la Paz, establece los instrumentos jurídicos de justicia transicional para la creación de una Jurisdicción especial para la paz, la cual empezará a funcionar una vez se firme el acuerdo final, y que tiene como finalidad establecer un mecanismo de justicia que satisfaga a las víctimas a su vez que juzgue a quienes hayan participado directa o indirectamente en el conflicto dentro de un marco de perdón sin que ello implique impunidad y contribuir así al logro de la paz estable y duradera que es el motor que impulsa este proceso, garantizando a las víctimas la justicia, verdad, reparación y garantía de no repetición. Lo anterior solo es aplicable a grupos del conflicto armado internos y que efectivamente ya estén desmovilizados.

Es por ello, que por medio de ley estatutaria se deberán establecer los delitos conexos al delito político, permitiéndole a las FARC los espacios para participar en política una vez comience el postconflicto, en todo caso dentro de estos no se podrán incluir los crímenes de lesa humanidad, genocidio y crímenes de guerra cometidos de manera sistemática [10].

Vale precisar que el Marco Jurídico para la Paz es aprobado y examinado constitucionalmente en el primer mandato del Presidente Juan Manuel Santos, y fue considerado como un ambiente preparativo para los nacientes diálogos con las FARC, proponiendo inicialmente, en digesto, que la paz es superior a la justicia, la igualdad y la libertad. Luego la Corte Constitucional, en sentencia C-579 de 2013 vigoriza este Marco con los derechos de las víctimas para que el sacrificio de la justicia no sea prominente.

En virtud de dicho Acto Legislativo el Gobierno nacional puede conceder indulto por este tipo de infracciones penales a ex combatientes de las FARC con el solo propósito de que puedan hacerse campo en la vida política del país de manera que, bien puede estar un ex guerrillero siendo procesado o haber sido condenado por transgresiones penales que tengan carácter 
de delitos políticos y conexos y aun así, seguir habilitado para ser congresista.

Asimismo, es de tener en consideración los alcances del indulto, ya que éste sólo suspende el cumplimiento de la pena para el caso de delitos políticos, evidenciando cierta incertidumbre en cuanto a los demás tipos de infracciones penales, en la medida en que una sentencia condenatoria que verse sobre modalidades delictivas que no tengan esta connotación especial imposibilitaría que este punto de la participación política pueda ponerse en marcha de manera exitosa [11].

Siendo así las cosas, es necesaria una regulación legal sobre amnistía y/o indulto (tenemos el ejemplo de la Ley especial de ocursos de gracia en el Salvador), que permita hacer compatibles la especialidad jurisdiccional para la paz con la participación de las FARC como un actor de la política nacional, atendiendo a los factores que hemos analizado.

Concepto de sistemas electorales y nociones básicas de sufragio, voto y escrutinio.

Aspectos esenciales al funcionamiento de una democracia son el sufragio, el voto y el escrutinio, elementos que se encuentran ineludiblemente relacionados y que deben coexistir para que el sistema democrático funcione a buen ritmo, el sufragio necesita del voto y los escrutinios no tendrían razón de ser si no hubiese una votación a contabilizar.

Ahora bien, cabe hacer una breve precisión en cuanto a lo que es el sufragio y en cuanto a lo que es el voto, pues es común asimilar estos dos términos cuando en realidad no son lo mismo; el primero se refiere al derecho que tiene todo ciudadano a optar por una elección política con la cual hacer afinidad, el derecho que tiene a elegir y a ser elegido; el segundo es la materialización del primero; el sufragio se concreta en la realidad a través del voto que se manifiesta en una papeleta, un tarjetón o por medios electrónicos [12].

En cuanto a los escrutinios vale decir que estos consisten en un examen de lo que han expresado los sufragantes en el ejercicio legítimo de la facultad que les ha sido otorgada de elegir la opción política que a bien consideren es la más apta para llevar las riendas del Estado. Tal análisis de los resultados de la contienda electoral se lleva a cabo implementando los sistemas electorales.

Los sistemas electorales son métodos de interpretación de resultados electorales que se valen de fórmulas y operaciones matemáticas preestablecidas. Cada sistema electoral trae su propia manera de traducir el producto de la votación obtenida en los comicios, y teniendo en cuenta que el futuro político del país se define en las elecciones escrutadas a través de uno u otro método de análisis, cada uno de estos traerá implícito un determinado interés, y utilizamos aquí las palabras del profesor David Mercado Pérez en su texto Manual de Teoría Constitucional [13]: "No existen sistemas electorales "neutros" ya que cualquier decisión sobre el sistema a adoptarse fortalecerá algunos grupos y limitará a otros".

Se evidencia aquí un aspecto que de entrada representa ciertos riesgos para los fines de la democracia, dado lo que ya hemos expresado sobre los sistemas electorales, se hará necesario que en consenso los distintos actores que intervienen en la política opten por aquel sistema que ajustándose al contexto histórico y socio-político les brinde a todos la garantía de que están ejerciendo de manera equitativa su derecho a ofrecerse como alternativa para gobernar el Estado. Tan problemático es este asunto que ha llegado a ser motivo de levantamiento de grupos subversivos, tal y como ha sido el caso colombiano. 
El sistema electoral de la cifra repartidora y la participación en política por parte de las farc en el marco del postconflicto colombiano

\section{0}

Un fenómeno interesante y que queremos traer a mención ahora es el Gerrymandismo, este debe su nombre a quien fue el responsable de idear toda esta estratagema para mantener su partido político perpetuamente en el poder Elbridge Gerry-Gobernador de Massachusetts y Vicepresidente de Estados Unidos bajo el mandato de Jamen Madison, se valió de una táctica por la cual se fraccionaba el territorio en donde se concentraba la votación de los afroamericanos, que si bien ya no estaban considerados como esclavos eran víctimas de una segregación extrema en la sociedad norteamericana, concentrando a su vez, la votación de su partido para que este ganara las elecciones, y así fue, entre 1863 y 1960 ningún miembro de la población afro logró ascender a cargos públicos pues la votación de esta población se diluía. Esta conducta hoy en Estados Unidos está prohibida, penalizada $y$ es causal de nulidad absoluta de las elecciones.

De manera que por Gerrymandismo entendemos la división estratégica del territorio en circunscripciones electorales con el propósito de manipular la votación de un determinado movimiento político, perjudicando entonces los resultados para este al tiempo que favorece a otro grupo político.

A continuación, explicaremos cada uno de estos sistemas electorales:

\section{1 - Sistema Mayoritario}

Conocido también como "la regla de la mitad más uno", este sistema está apoyado en el criterio de que en una democracia la minoría que ha resultado vencida en la competencia por el voto popular deberá entonces someterse a las decisiones de la mayoría que ha resultado ganadora en esta contienda.

Opera mediante una serie de rondas en las que se van eliminando a los postulantes que obtengan las menores votaciones, lo que se conoce como "Sistema de doble vuelta" - BALLOTAGE. Colombia lo utiliza en las elecciones presidenciales.

Para su operancia es necesario que se cumplan ciertas condiciones o presupuestos para que este no termine desbordándose y convirtiéndose en un atentado contra la democracia:

- Que solo se use en una sola circunscripción electoral.

- Que solo compitan dos partidos políticos en dicha circunscripción única.

- Que compitiendo solamente dos partidos, en una sola circunscripción, ninguno de ellos se divida [14].

\section{2 - Sistema Minoritario}

Llamado también de "voto incompleto", por ley se establecen cuantas curules corresponden al ganador y cuantas al perdedor. Solo se tienen en cuenta las mayores votaciones por lo que es funcional sólo si existen dos partidos; si existen más, el sistema falsea los resultados y pierde credibilidad democrática.

Este fue el sistema que se implantó en Colombia durante el Frente Nacional, siendo el factor que dio origen a las guerrillas, y que posteriormente, aun habiéndose abolido el Frente Nacional en lo que respecta al ejecutivo se siguió implementando, puesto que los partidos liberal- conservador hicieron un pacto por el cual equitativamente el partido perdedor gobernaba junto al partido del presidente.

Es con el gobierno de Virgilio Barco Vargas que este entra en crisis, pues decide darle la oportunidad a los guerrilleros desmovilizados del M-19, ERL y EPL de participar en la política colombiana, de manera que el movimiento conservador decidió a manera de protesta no 
aceptar cargos públicos y conformarse con tener mayorías regionales, aquí empieza su decadencia, y es con Álvaro Uribe que este logra tomar cierta fuerza nuevamente en la arena política del país.

\section{3 - Sistema de Representación Proporcional}

Su nombre nos dice su intención, evitar las desproporciones en la distribución del poder.

\section{Clasificación de las fórmulas electorales}

a) Cociente Electoral o Fórmula de Haré. Fue la que existió en Colombia para elecciones al senado, Cámara de Representantes, Asambleas Departamentales, Concejos municipales y Juntas Administradoras locales. Aplicándose hoy para determinación del "Umbral" o votación mínima. Este sistema funciona así:

- Se suman los votos depositados en las urnas de la respectiva circunscripción electoral más los votos en blanco y se divide el resultado de la misma entre el número de curules o escaños en disputa. El resultado de esta es el Cociente electoral.

- Se verifica cuantas veces esta repetido el cociente electoral en la votación de cada lista o plancha.

- Cada vez que el cociente electoral esté contenido en forma entera significa que la respectiva lista habrá obtenido una curul.

- Agotado el procedimiento anterior, si quedasen curules por asignar se utilizara el residuo electoral. Se llama así a la diferencia entre el total de votos obtenido en cada lista y el total de votos que en forma entera contenga el cociente electoral. Si alguna lista obtuvo una votación inferior al cociente electoral, dicha votación se mirará toda como un residuo.
- Las curules sobrantes se le adjudican a las listas con residuos más altos, una por una, hasta que no quede ninguna [14].

En esta operación el divisor trabajo como constante, y dividendo y cociente actúan como variables directamente proporcionales, a medida que el cociente aumenta también van aumentando los residuos, de manera que se adjudicarán más curules por estos que por el mismo cociente.

Es psoible percatarse entonces de la trampa legal que trae el voto en blanco, puesto que contrario a su finalidad este no viene a tomarse como una expresión de inconformidad con las opciones políticas ofrecidas sino que se suma a la fórmula para el conteo e interpretación de los resultados y asignación de escaños, es decir, los votos en blanco van a ayudar a inflar las votaciones y si tomamos en cuenta todo lo explicado tenemos un sistema que propicia el clientelismo y la perpetuación en el poder de las hegemonías políticas que históricamente lo han detentado en nuestro país, a su vez que tiende a asfixiar a los movimientos minoritarios.

Es lo que sucedió con la llamada "operación avispa" ingeniada por el doctor ALFONSO LOPEZ MICHELSEN, que observando el panorama político de cara a unas elecciones para la Asamblea Nacional Constituyente $y$ ante una alta posibilidad de pérdida del partido liberal, sugirió que este se presentara no como una única lista sino como varias listas, de manera que con ayuda de expertos matemáticos convocados por el presidente Cesar Gaviria (liberal) se estableció el número de listas necesarias para obtener más curules que el M-19 que se mostraba como favorito.

b) Fórmula de Droop. Se suma una unidad imaginaria a las curules a asignar, buscando que el cociente disminuya y así también sea menor el número de escaños a fijar por residuo. 
El sistema electoral de la cifra repartidora y la participación en política por parte de las farc en el marco del postconflicto colombiano

92

Aun así, si el partido ganador obtiene una votación que supere en dos veces y media la del perdedor aparece la trampa. Con la reforma de 1968 y hasta la Constitución de 1991 se implementó esta figura.

c) Fórmulas del Divisor. Buscan asegurar el reparto de curules por medio de la división de la votación de cada partido entre series de divisores, con los que el reparto se hace por la media aritmética mayor, por lo que los residuos electorales no se necesitan.

Son estas fórmulas las siguientes:

\begin{tabular}{ll}
\hline MÉTODO & DIVISORES \\
\hline D'HONT & $1,2,3,4,5$, etc. \\
\hline SAINT LAGUÉ & $1,3,5,7,9$, etc. \\
\hline SAINT LAGUÉ modificada & $1,4,3,5,7$, etc. \\
IMPERIALI & $2,3,4,5,6$, etc. \\
\hline DANÉS & $1,4,7,10,13$, etc. \\
HUNTINGTON & $1-1,5-2-2,5-3-3,5-4-4$, etc. \\
\hline
\end{tabular}

Todas estas fórmulas operan en lo matemático de idéntica manera:

- La votación de cada partido se divide por los divisores que previamente se hubiesen adoptado.

- Se determinan los cocientes que cada partido obtenga y luego se verifican los más altos, por cada uno de estos, cada partido consigue un curul o escaño [14].

- Los votos obtenidos por partido vienen a ser el dividendo, de manera que este será invariable y son los divisores los que cambian y dividendo y cociente son entonces inversamente proporcionales. A medida que aumenta el divisor se va haciendo menor el cociente y siendo así son más partidos los que tienen opciones de obtener curules por este.

Sucede algo interesante con esto y es que si los divisores son menores (D'HONT) se beneficia a los partidos con mayor votación y si son mayores se beneficia a los que tienen menos votos.

Así las cosas, estas fórmulas lo que propician es una redistribución de las bancas políticas. Hay una desproporción de una formula a otra, lo que señalábamos al principio, los sistemas electorales no son neutros.

La cifra repartidora en colombia y participación política de la alternativa al poder

Por medio de Acto legislativo 01 del año 2003 se modificó el sistema de asignación de curules en las corporaciones públicas de la fórmula del cociente electoral a la cifra repartidora, esta 
consiste entonces en el número mínimo de votos que debe obtener cada partido político para poder lograr una curul, y funciona de la siguiente manera:

- El número de votos obtenidos por cada lista se divide sucesivamente entre uno, dos, tres o más.

- Se ordenan los resultados de forma decreciente hasta obtener un número total de resultados igual al número de curules a proveer. Al menor resultado se le llama Cifra repartidora.

- El número de curules obtenido será el número de veces que la Cifra repartidora este contenida en el total de votos obtenidos por lista.

Si bien esta fórmula en un principio se adopta con la intencionalidad de distribuir de manera equitativa los escaños en las corporaciones públicas y garantizar la igualdad de condiciones en materia de participación a los diferentes movimientos políticos, la verdad es que este sistema es peligroso para la democracia, en la medida en que se complementa con el umbral o votación mínima, pues propende por que los partidos mayoritarios sigan repartiéndose el poder entre sí y tiende a la desaparición de los partidos minoritarios, propiciando un ambiente de alianzas perversas entre unas y otras fuerzas políticas en un afán desesperado por sobrevivir.

En realidad, la Cifra Repartidora es una fórmula de D'Hondt disfrazada porque usa como divisores la serie de los números naturales y privilegia a los partidos con votación más alta ya que obtienen un porcentaje de escaños mucho más alto que el porcentaje de curules. Todo ello "complementado" con el Umbral basado en el Cociente Electoral que admite distorsiones como la "Operación Avispa", de modo tal que quien no lo supera no entra en el reparto de Curules.

Una sociedad democrática y pluralista como la colombiana no solo tiene en cuenta las decisiones de la mayoría sino que aprecia las opiniones minoritarias, permitiéndoles espacios para expresarse libremente en un marco de respeto y tolerancia. Los partidos políticos son expresión de distintas perspectivas y enfoques sociales y pretenden ejercer el poder político del Estado de manera coherente y acorde con el discurso que profesan y gobernar a partir de esas ideas.

La democracia en este sentido, busca que todas las organizaciones políticas tengan las mismas posibilidades de aspirar y llegar al poder, mediante una competencia abierta y transparente por el voto de los ciudadanos. En este punto los medios de comunicación juegan un papel fundamental, puesto que es a través de ellos que el sufragante interactúa con la imagen de los distintos candidatos a los cargos de elección popular.

La elección dependerá de la imagen que se muestre y se exhiba de los postulantes de manera que, las intenciones de llegar al poder se supeditan a la neutralidad e imparcialidad de los medios y a espacios igualitarios para difundir sus propuestas, los partidos políticos se harán de estrategias publicitarias para ofrecer a sus aspirantes como la mejor alternativa y lograr mantenerse vigentes.

Por lo anterior, y atendiendo a las exigencias de las FARC se hace menester que para personificar su participación en política (lo cual implica permitirle al partido o movimiento político que constituyan llegar a los órganos del Estado y ser una alternativa del poder) debe darse una profundización de la democracia participativa eliminando la cifra repartidora e implantando la fórmula de saintlagué o una 
El sistema electoral de la cifra repartidora y la participación en política por parte de las farc en el marco del postconflicto colombiano

\section{4}

saintlagué modificada, y así lograr erradicar las manipulaciones en los escrutinios.

Los riesgos del acuerdo y la inclusión de una democracia directa

Laparticipación política de las FARCy la idea de democracia directa que introduce y desarrolla el acuerdo, pone de presente un concepto de democracia para ser ejercida más allá de los partidos políticos. La postura institucional apoya que los partidos y sus representantes elegidos por el voto popular son el instrumento que permite representar los intereses de los ciudadanos en el escenario público, las Farc defienden la idea que los movimientos sociales son mucho más representativos de los intereses del pueblo que los partidos políticos y unas de sus reivindicaciones en la mesa eran que las garantías de un eventual Estatuto para la Oposición se hicieran extensivas a las movilizaciones sociales. El Estatuto de la oposición se debatirá entonces con los partidos políticos una vez se llegue a un Acuerdo de Paz.

El acuerdo plantea varias garantías para la participación política directa de los movimientos sociales, una Ley de Garantías, la posibilidad de que los movimientos sociales den a conocer sus propuestas en medios institucionales y regionales, la posibilidad de que formen parte de Veedurías Ciudadanas que ejerzan control frente a los gobernantes, la posibilidad de que participen en la elaboración y seguimiento a los planes de desarrollo local, integrando los Consejos Territoriales de Planeación y apoyos especiales a nuevos movimientos y partidos políticos. Sin embargo, uno de los riesgos de la inclusión de una democracia directa en el Acuerdo, la encontramos en las Circunscripciones Transitorias Especiales de Paz. Estas circunscripciones buscan integrar a las regiones más golpeadas por el conflicto y es un mecanismo que podría favorecer a las Farc en la consecución de una mayor representación política de sus bases en el Congreso.

Básicamente, en lo que consisten estas circunscripciones es en permitir que en estas regiones, y durante un período de tiempo determinado, se puedan elegir "representantes adicionales con unas reglas especiales". Esto le abre la posibilidad a las Farc para tener un caudal político de entrada a la vida civil si es que realmente demuestran que tienen bases sociales que creen en sus ideas y que votan por ellos sin la presión de las armas. Así las cosas, el acuerdo logrado es muy beneficioso para ellos pues podrían modificar desde "abajo" el mismo sistema electoral ya que la participación de la gente sería mucho más directa en asuntos de planeación, de veeduría $y$ de quienes llegan al Congreso [14].

\section{Conclusiones}

Se hace evidente que las FARC buscan en la Mesa de diálogos tener un espacio político en el sistema y ser alternativa política válida de gobierno. La derecha tradicional debe compartir el poder o bien soportar el desgaste de la lucha guerrillera y de las organizaciones armadas del narcotráfico, algo impensable en la era de la globalización.

Con lo acordado en el punto segundo de la agenda se dan pasos importantes para lograr una participación en política por parte de las FARC con garantías que quedarán normadas en el Estatuto de la Oposición, y se harán palpables con el Sistema Integral de Seguridad para el ejercicio de la política, además de la creación de las Circunscripciones Transitorias Especiales de Paz en las regiones más golpeadas del conflicto armado y el establecimiento de del Consejo Nacional para la Reconciliación y la Convivencia, para la promoción de la cultura de paz, tolerancia y reconciliación. 
Para evitar castrar las posibilidades políticas de otras fuerzas sociales, debe desmontarse la fórmula del cociente electoral que permite la manipulación técnica de las votaciones, haciendo que los residuos tengan un amplio margen y las "operaciones avispas" ignoren la voluntad popular. No hay que olvidar que esta fórmula es la que aplica para determinar el "Umbral".

También se hace necesario la expedición de una ley de amnistías e indultos que permita hacer compatibles la especialidad jurisdiccional para la paz con la participación de las FARC como un actor de la política nacional.

\section{Referencias}

[1] Acuerdo General para la terminación del conflicto y la construcción de una paz estable y duradera, suscrito el 26 de agosto de 2012 en la Habana, Cuba.

[2] COLOMBIA. CORTE CONSTITUCIONAL. Sentencia C- 579 de 2013. Magistrado Ponente: Dr. Jorge Iván Pretelt Chaljub.

[3] D. Mercado, "La paz en Colombia: marco histórico, político y jurídico. Revista De La Asociación Colombiana De Técnicas De La Industria De La Pulpa, Papel Y Cartón", ACOTEPAC, vol. 1, 1998

[4] D. Mercado, Manual de Teoría Constitucional. Rodríguez Quito Editores, 2008

[5] U. Rodrigo y otros, "Justicia Transicional y Proceso de Paz en Colombia", Revista Aportes DPLF, no. 18, año 6, Diciembre de 2013

[6] I. Blanco Rangel, "Apuntes sobre Colombia a propósito de su internacionalización y otros desafíos", Aglala, vol. 7, no. 1, pp. 209-224, 2016

[7] A. Caballero Tovío, "Grado de articulación de las estrategias consignadas en el Plan Nacional de Desarrollo 2014-2018 con la situación del sector industrial colombiano", Enfoque Disciplinario, vol. 1, no. 1, pp. 14-20, 2016

[8] O. Bernal Payares, "Evolución del proceso administrativo desde los orígenes de la revolución industrial y su fundamento como una función del administrador", Enfoque Disciplinario, vol. 2, no. 1, pp.1-14, 2017

[9] I. Satizábal, "Seguridad de los protocolos de voto electrónico a través de internet; una comparación", Revista Tecnología de Avanzada, vol. 2, no. 26, 2015

[10] A. Aguilar Barreto, C.Y. Soto Rojas y J.M. Castaño Quijano, "Políticas públicas de juventud: factor de desarrollo y participación en Norte de Santander y San José de Cúcuta", Mundo Fesc, vol. 5, no. 10, pp. 69-74, 2016.

[11] M. Peña, "Anuario Colombiano de Historia Social y de la Cultura", Anu. colomb. histo. soc. cult, vol. 41, no. 1, pp. 201-236, 2014

[12] L. Trejos, "Colombia: una revisión teórica de su conflicto armado", Revista Enfoques, vol. XI, no. 18, pp. 55-75, 2013

[13] F. Suarez Vargas, J.A. Gómez Montoya y L. Ballesteros Arenas, "Eficacia en la restitución de tierras a víctimas de desplazamiento forzado de Ocaña Norte de Santander", Mundo Fesc, vol. 5, no. 10, pp. 61 - 68, feb. 2016

[14] M. Luna Benítez, "El M-19 en el contexto de las guerrillas en Colombia". Sociedad y economía, vo. 10, pp. 157-188, 2006 